\title{
A Joint Statement for "Tighter Regulation" of Traditional Chinese Medicine Issued by the Federation of European Academies of Medicine (FEAM) and the European Academies' Science Advisory Council (EASAC): Scare Stories or Obstruction of Access?
}

\author{
Dieter Melchart \\ Center of Complementary Medicine and Naturopathy (COCONAT), Faculty of Applied Healthcare Sciences, \\ Technische Hochschule Deggendorf, Munich, Germany
}

Traditional Chinese Medicine (TCM) with its focus on herbal use is popular and appreciated worldwide with increased tendency, although its therapeutic efficacy is still not enough established for most TCM procedures. Treatment is perceived as fairly safe; however, eager discussions have emerged more recently as to whether herbal TCM and other procedures like acupuncture have undesired side effects. A joint statement for more and stronger regulation of TCM issued by the Federation of European Academies of Medicine (FEAM) and the European Academies' Science Advisory Council (EASAC) punctuates this trend $[1,2]$.

The statement is related to the recent revision of the International Classification of Diseases coding tool (ICD11) by the World Health Organization (WHO). The WHO decided to add a chapter on TCM to the ICD-11, which lists treatments globally available for a variety of medical conditions. It contains a list of categories, including "26 Supplementary Chapters of Traditional Medicine Conditions - Module I" [3]. ICD-11 was released in June 2019 for adoption by Member States and would come into effect in January 2022.

Now, there is concern that this inclusion "may contradict the scientific principles on which ICD has been built" [1]. There is also fear that this recognition will encourage the use of unproven therapies and the public might be "misled into thinking there is good evidence for TCM products and procedures and that they work and are safe" [1]. Further, this might promote reimbursement by public health systems at a time of limited resources.

There is strong agreement among scientists and practicing doctors that all medical remedies, no matter if they belong to classical or complementary treatment strategies, have to be evaluated appropriately for their benefit, safety, and cost-effectiveness, consistent with adequate testing procedures. However, it is wrong to assume that TCM treatment or diagnostic procedures per se are not evidence based, while all mainstream medical procedures are.

Further, the statement explains that "TCM is an integral part of health services in some Asian countries, but although there has been some convergence there is no agreed international standard to allow collection of comparable data between countries and no common starting point for testing efficacy of interventions or monitoring safety." Moreover, "In Europe it is difficult to reconcile TCM with mainstream medicine and its frameworks for regulation" [2].

So, it remains unclear why EASAC complains about missing international standardization in TCM on the one 
hand, while fighting against any inclusion of TCM in the coding system on the other. The integration of Chinese medical terminology into the ICD coding system could strongly improve international education, practice, standardization, safety, and academic exchanges of Chinese medicine.

It is also elusive why the European bodies blame the concept of TCM diagnostic to be merely based on symptoms and signs. The existing ICD-10 coding system comprised several chapters like R00-R99 including symptoms, signs, abnormal results of clinical procedures, and ill-defined conditions for which no diagnosis classifiable elsewhere is recorded. Further, Z00-Z99 category is used when some circumstance or problem is present which influences the person's health status but is not in itself a current illness or injury.

On the contrary, the diagnostic categories of TCM describe clinical patterns of both subjected symptoms and visually objective signs but simultaneously enable treating patients according to these unique patterns of dysfunction and emphasis on restoration of normal physiological functioning. The identification of diagnostic patterns guides practitioners to clearly defined therapeutic approaches in treatment and prevention, using acupuncture, herbal medicine, dietary changes, or other procedures [4]. Chinese herbal medicine works hand in hand with the concept of patterns of dysfunction. For this reason, the fundamental principle is to restore balance and harmony: when the patient's pattern is hot, cool it; when cold, warm it; when deficient, tonify it, when dry, moisten it [4]. The treatment principles here are antagonistic.

Patients with a distinct Western diagnosis might get different treatments in TCM while, on the other hand, patients with different Western diagnoses might get the same treatment. In this way, TCM adds more treatment options for people and can be considered as complementary to conventional Western practice in our European countries. This can be regarded as extremely beneficial, particularly in cases where conventional therapy fails and whenever a non-mainstream practice is used together with (and not in place of) conventional medicine, either side by side or one after the other.

Furthermore, TCM herbs might turn up being useful components, as many Western drugs derived from plants. But instead of using these opportunities, there is more worry that TCM could replace proven Western drugs, increase pressure for reimbursement by public health systems, and may bring additional health risks [2]. With respect to herbal medicines, the FDA classifies them as "dietary supplements," as it does with all botanical medicines. Thus they are regulated under the Dietary Supplement Health and Education Act (DSHEA). Manufacturers of TCM herbs do not need to get premarket approval from the FDA, but they are responsible for determining that their products are safe and that their product claims are proven by evidence. As long as TCM herbs lack, e.g., inconsistent quality of the herbal material and ethical committees do not allow to prove them, it is unlikely that there is much intention by pharmaceutical companies to invest money in clinical research, like Western companies do, with legal patents.

Results from a number of studies suggest that acupuncture may help relieve several types of pain that are often chronic such as low back pain, neck pain, and osteoarthritis/knee pain [5-7]. It also may help reduce the frequency of tension headaches and prevent migraine headaches [8]. Therefore, acupuncture appears to be a reasonable option to consider for people with chronic pain and sometimes offers an alternative to drug therapy like opioids. These drugs are well evidenced but not free of harm: more than 130 people died every day from opioid-related drug overdoses in 2016 and 2017, according to the US Department of Health and Human Services. Beyond that, Chinese medicine may have much to offer in terms of preventing illness and disease, optimizing our health, and enhancing our well-being.

It has become obviously fashionable by FEAM and EASAC to disclaim therapeutic efficacy of complementary medicine in view of a lack of plausibility, although some published evidence suggests the opposite [9-12]. Is it telling scare stories in a sense of a polemic propaganda or ignorance against the most commonly used complementary medicine concept worldwide? The broad range of individual needs and preferences of the European population requires more complementary medical choices, even though it has to be proven and safe. However, patients' preferences and values are a very important matter in evidence-based medicine and for any political decision-making. That means simply a call for more pluralism in European medicine.

It is not fair to claim highest evidence from a part of medicine which has not yet received enough support to be established on international scientific floors - but TCM shows reasonable signs to fulfil these postulations [13]. In the recent 3 years (from 2015 to 2017), the number of registered TCM trials has grown rapidly, which accounted for $51 \%(1,701 / 3,339)$ of all TCM registered trials up to the end of 2017. Most of the TCM trials $(2,805 / 3,339)$ were conducted in a randomized format, although the majority of them had less than 100 subjects enrolled [13]. $42.6 \%$ of all TCM clinical trials were performed and registered outside the mainland of China, e.g. 9.2\% (308 studies) in the USA. But clinical evidence from studies alone is never sufficient for decision-making. It has to be integrated with clinical expertise and patients' expectations and values.

Chapter 26 has been designed as a standard reference that all practitioners can use for describing TCM diagno- 
ses. The chapter is for optional use only and not intended for mortality or morbidity reporting. Coding of diseases will still be made according to the other chapters already established in ICD-10. In contrast to the other chapters, chapter 26 can also be used for research in the field of traditional medicine such as the quantification of adverse events and the interaction between herbal TCM and Western pharmaceutical drugs. It might help to promote the safe and effective use by developing proper quality standards and regulations to ensure a similar safety standard as Western drugs [13]. Hopefully, these objectives are on behalf of both, the WHO and the FEAM.

\section{References}

1 https://www.theguardian.com/education/2019/nov/06/doctors-europe-call-fortighter-control-of-traditional-chinesemedicine?CMP=Share_iOSApp_Other

2 https://easac.eu/fileadmin/PDF_s/reports_ statements/Traditional_Chinese_Medicine/ EASAC-FEAM_TCM_statement_final_ Nov_2019.pdf

3 https://icd.who.int/browse11/l-m/en

4 Bensoussan A, Myers St P. Towards a Safer Choice. The practice of Traditional Chinese Medicine in Australia. Faculty of Health, University of Western Sydney Macarthur; 1996. pp. 24-5.

5 Vickers AJ, Linde K. Acupuncture for chronic pain. JAMA. 2014 Mar;311(9):955-6.

6 Witt CM, Jena S, Brinkhaus B, Liecker B, Wegscheider K, Willich SN. Acupuncture in patients with osteoarthritis of the knee or hip: a randomized, controlled trial with an additional nonrandomized arm. Arthritis Rheum. 2006 Nov;54(11):3485-93.

7 Berman BM, Langevin HM, Witt CM, Dubner R. Acupuncture for chronic low back pain. N Engl J Med. 2010 Jul;363(5):454-61.

8 Linde K, Allais G, Brinkhaus B, et al. Acupuncture for migraine prophylaxis. Cochrane Database of Systematic Reviews. 2009;(1): CD001218. Accessed at www.thecochranelibrary.com on July 2, 2014.

9 Liang X, Li H, Li S. A novel network pharmacology approach to analyse traditional herbal formulae: the Liu-Wei-Di-Huang pill as a case study. Mol Biosyst. 2014 May;10(5): 1014-22.

10 Chao J, Dai Y, Verpoorte R, Lam W, Cheng YC, Pao LH, Zhang W, Chen S. Major achievements of evidence-based traditional
Chinese medicine in treating major diseases. https://doi.org/https://doi.org/10.1016/j. bcp.2017.06.123.

11 Chen H, Wang Y, Jiang WB, Kwong JS, Gu YH. The evidence system of traditional Chinese medicine based on the Grades of Recommendations Assessment, Development and Evaluation framework. Ann Transl Med. 2017 Nov;5(21):435.

12 Manheimer E, Wieland S, Kimbrough E, Cheng K, Berman BM. Evidence from the Cochrane Collaboration for Traditional Chinese Medicine therapies. J Altern Complement Med. 2009 Sep;15(9):1001-14.

13 Zhang X, Tian R, Yang Z, Zhao C, Yao L, Lau $\mathrm{C}$, et al. Quality assessment of clinical trial registration with traditional Chinese medicine in WHO registries. BMJ Open. 2019 Feb; 9(2):e025218. 